\title{
Illegal drug use in the age of ageing
}

Both historically and currently, UK policies on illegal drug use have been largely focused towards younger people, but there is growing evidence of a shift in the demographic profile of people who use illegal drugs, with an increasing substantial number of drug users aged over 40 years. There is limited evidence for a change in the age profile of drug users from the British Crime Survey which documents drug use in the general population of England and Wales. ${ }^{1}$ This survey reports that the prevalence of illegal drug use (predominantly cannabis, cocaine powder, ecstasy, amyl nitrate, and amphetamines) in the previous year has fallen significantly between 1996 and 2008/2009 for those aged 16 to 29 years, while the prevalence has increased among those aged 30 to 59 years. ${ }^{1}$ Unfortunately, the British Crime Survey does not collect information about illegal drug use by those aged 60 years and over, so identifying population level data on drug use for the oldest age groups presents a challenge.

Further evidence of this demographic change among people accessing drug treatment services (primarily users of opiates and/or crack cocaine), ${ }^{2}$ is available from surveillance data. Using data from established monitoring systems (including the National Drug Treatment Monitoring System [NDTMS] which currently records data on people accessing structured treatment interventions in England), Beynon et $a^{3}$ demonstrate that the percentage of people in drug treatment in Cheshire and Merseyside aged 40 years and over increased from $9.8 \%$ in 1998 to $23.1 \%$ in 2004/2005 (with $1.7 \%$ of these aged 50 years and over in 1998 compared to $3.5 \%$ in $2004 / 2005$ ).

More recently, data from the NDTMS for the North West region of England (which encompasses Cheshire, Merseyside, Greater Manchester, Cumbria, and Lancashire) show the percentage of people accessing drug treatment in 2008/2009 who were aged 40 years and over rising further to $30.8 \% .^{4}$ In
2008/2009 there were 210815 patients aged 18 years and over in treatment in England; ${ }^{2}$ if we extrapolate the age distribution of the North West, 64931 of these will be aged 40 years and over.

That drug users are living longer is encouraging. However, a recent 5-year study about the deaths of 504 drug treatment patients shows that they still die prematurely. While the median age at death increased significantly, by almost 5 years between 2003/2004 and $2007 / 2008$, the median age at death in $2007 / 2008$ was still only 41.4 years (interquartile range $36.6-48.7$ years). ${ }^{5}$ Furthermore, this study reported that people aged 40 years and over are dying of different causes than people aged less than 40 years. More specifically, only $22 \%$ of deaths among those aged 40 years and over were classified as a drug-related death (that is, from the acute effects of drug use; mainly mental and behavioural disorders and poisoning), with liver diseases, neoplasm, chronic lower respiratory infections, and viral hepatitis accounting for the largest number of deaths from non-acute causes. ${ }^{5}$

Drug users aged 40 years and over therefore experience different health challenges as they age, and the reasons for this are numerous. First, longer duration of drug use means a longer window of exposure for certain disease risk factors; for example, longer duration of injecting is associated with an increased risk of contracting hepatitis C. ${ }^{6}$ Second, long-term use of drugs, often in association with the use of alcohol and tobacco in this population, ${ }^{7}$ is associated with biological and physiological changes which increase the risk of certain conditions, such as chronic obstructive pulmonary disease and other chronic respiratory diseases. Third, it is a well accepted phenomenon that the least advantaged in society make the fewest demands on specialist health services ${ }^{8}$ and drug users are, therefore, unlikely to take full advantage of preventative screening programmes. Consequently, certain types of neoplasm, for example, are unlikely to be detected as early in drug users as in other sections of society. Finally, macro determinants of health, in particular the impact of long-term deprivation, cannot be ignored. Whatever the specific cause, many people aged 40 years and over who are long-term users of illegal drugs are biologically much older than their chronological age.

The burden of conditions associated with long-term drug use should not be underrated. It is estimated that there are around 185000 individuals with chronic hepatitis $\mathrm{C}$ infection in the UK; around $90 \%$ of these have a history of current or past injecting drug use. Although the rate of testing of those in treatment is improving, there is a 'silent reservoir' of infection in users or ex-users, the majority of whom will not be in treatment but who may be accessible through primary care. ${ }^{9}$ We have no information of the burden of other long-term conditions among current and ex-drug users but this will only increase as more reach older age.

How should we respond? Firstly, and most importantly, this issue requires heightened awareness among both the general public and health professionals. Primary care and other health services provide an opportunity to identify drug use, but work in the US demonstrates that doctors are currently poor at diagnosing the abuse of alcohol among older people,$^{10}$ let alone diagnosing the use of illegal drugs which we presume is mainly associated with younger people.

Some people recommence or escalate drug use in later life, ${ }^{11}$ so general practice should be vigilant to the possibility of drug use across a person's life course. Any previous history of drug use may be highly significant but in the older person, further cues may not be obvious and, given the disenchantment some have experienced with health services, substance use beyond alcohol and tobacco may not be volunteered without specific enquiry. It would be appropriate to consider drug use in anyone presenting with relatively premature chronic disease, particularly of the lungs and liver, and there may be an 
increase in alcohol consumption in some ageing users as their pattern of substance misuse shifts.

In those who are identified, appropriate harm reduction advice is necessary so that older people understand the interactions between drug use and ageing; for example, deep vein thrombosis may become more prevalent among older injectors, particularly among those who smoke, because injectingrelated damage will be exacerbated by age-related changes. Clinical vigilance will be required to identify early onset chronic obstructive lung disease and other respiratory diseases prevalent among those who smoke crack cocaine, heroin, cannabis, or tobacco. Similarly, stimulant and opiate-related cardiopathology and renal disease will require identification, monitoring, and management. In short, primary care clinicians need to screen for, and treat, the chronic health conditions among users of illegal drugs as well as simply treating their drug use. ${ }^{12}$

However, the care of older drug users presents unique challenges. The use of prescription drugs, which increases in older age, particularly among those with poor health, and the interactions of these with illegal drugs need consideration, as will the possibility of iatrogenic addiction to prescribed medications. Drug and alcohol-related cognitive impairment, which is likely to occur at a relatively young age, ${ }^{11}$ may make treatment adherence a problem. Pain management and palliative care will become important issues as a growing number of drug users reach older age. Opiate users have more complex needs than the general population, and concern about practitioners providing inadequate analgesia, particularly to those patients receiving methadone as part of substitution therapy, has already been raised. ${ }^{11}$

Evidence showing that older drug users have little or no family support ${ }^{11}$ suggests that social care in addition to health services will be necessary, and the reality we face is rising costs associated with caring appropriately for the increasing number of drug users reaching older age. Only now are we becoming aware of the challenge this demographic change presents.

\section{Caryl Beynon,}

Reader in Substance Use Epidemiology, Centre for Public Health Research Directorate, Faculty of Health and Applied Social Sciences,

Liverpool John Moores University.

\section{Gerry Stimson,}

Executive Director, International Harm

Reduction Association, London.

\section{Euan Lawson,}

GP Specialist in Substance Misuse, Delphi

Medical Consultants, Lancaster.

\section{Provenance}

Commissioned; not peer reviewed.

\section{REFERENCES}

1. Hoare J. Drug misuse declared: findings from the 2008/09 British Crime Survey. London: Home Office; 2009.

2. Department of Health and the National Treatment Agency. Statistics from the National Drug Treatment Monitoring System (NDTMS) 1 April 200831 March 2009.

http://www.medicine.manchester.ac.uk/healthmethod ology/research/ndec/factsandfigures/NDTMSstatistics/ (accessed 15 Jun 2010)

3. Beynon CM, McVeigh J, Roe BA. Problematic drug use, ageing and older people: trends in the age of drug users in northwest England. Ageing Soc 2007; 27(6): 799-810.

4. Hurst A, Marr A, McCoy E, et al. Drug and alcohol treatment in the North West of England 2008/09. Results from the National Drug Treatment Monitoring System (NDTMS). Liverpool: Centre for Public Health, 2010.
5. Beynon C, McVeigh J, Hurst A, Marr A. Older and sicker: changing mortality of drug users in treatment in the North West of England. Int J Drug Pol 2010 Feb 19. [Epub ahead of print].

6. Hope VD, Judd A, Hickman M, et al. Prevalence of hepatitis $\mathrm{C}$ among injection drug users in England and Wales: is harm reduction working? Am Journal Public Health 2001; 9(1):38-42.

7. Department of Health (England) and The Devolved Administrations. Drug misuse and dependence: UK guidelines on clinical management. London: Department of Health (England), the Scottish Government, Welsh Assembly Government and North Ireland Executive, 2007.

8. Veugelers P, Yip A. Socioeconomic disparities in health care: Does universal coverage reduce inequalities in health? J Epidemiol Community Health 2003; 57(6): 424-428.

9. Health Protection Agency. Hepatitis $C$ in the UK. London: Health Protection Agency, 2009.

10. Boddiger D. Drug abuse in older US adults worries experts. Lancet 2008; 372(9650): 1622.

11. Beynon CM, Roe B, Duffy P, Pickering L. Self reported health status, and health service contact, of illicit drug users aged 50 and over: a qualitative interview study in Merseyside, United Kingdom. BMC Geriatr 2009; 9: 45.

12. Han B, Gfroerer JC and Colliver JD. Associations between duration of illicit drug use and health conditions: results from the 2005-2007 national surveys on drug use and health. Ann Epidemiol 2010; 20(4): 289-297.

DOI: 10.3399/bjgp10X514710

\section{ADDRESS FOR CORRESPONDENCE}

\section{Caryl Beynon}

Centre for Public Health Research Directorate, Faculty of Health and Applied Social Sciences, Liverpool John Moores University, 4th Floor Kingsway House, Hatton Garden, Liverpool L3 2AJ. Email: c.m.beynon@ljmu.ac.uk 